\title{
CLOUD COMPUTING APPLICATION AND SECURITY IN SMART POWER GRIDS
}

\author{
Ayushi Ghill, Anshita Dharmawat, Yogesh Shirmali
}

E-Mail Id: ayudhanu17@gmail.com, ansh.dharmawat@gmail.com'yogeshshrimali1@gmail.com Department of Computer Science \& Engineering, Aravali Institute of Technical Studies, Udaipur, India

Abstract- with the appearance of the reforms of the third era, people's lives have undergone tremendous changes, and \{also the \}ower development has also accelerated. The sensible facility is wide used, and supported its specific options, it's developed a cloud computing technology with additional convenient, quick calculation strategies and storage modes. This paper proposes specific cloud computing solutions to supply effective technical support for storage and computing of sensible power grids. At a similar time, we have a tendency to style a theme for sensible facility cloud computing, and explains the varied edges with the cloud computing, and therefore the existing security problems and preventive measures are mentioned.

Keywords: component; smart power grid; cloud computing; security

\section{SMART POWER GRID RELATED DESCRIPTION}

The good grid will collect the relevant data concerning the grid operation within the shortest time, and might timely analyze the system knowledge through its own automation perform within the shortest time to create the simplest response set up. The good power system will be intelligent and refined supported an oversized quantity of knowledge. It directly bears all tasks of the electrical output. It links the user and also the relevant departments of the ability grids through the intelligent terminal, in order that the info assortment will be timelier and quicker, that essentially improves the applying potency of the ability grid. the aim of the good power system is to complete the energy substitution and attain the goal of energy saving and emission reduction, \{the data|theinfo|the data\} are going to be fully sorted out and also the grid are going to be optimized and updated supported information assortment and system institution. In fact, this understanding of good power grids has nice limitations. the general public assume that good power system may be a comprehensive tool, which might complete commands in line with the large knowledge it's, like self- defense throughout attack and keep the grid operating.

\section{SMART POWER GRID FEATURES AND RISKS}

\subsection{Smart Power Grid Features}

The main characteristics of the sensible installation square measure self- healing, safe, high compatibility and sensible coordination. Self- healing is once the sensible installation has issues, the relevant instrumentation can create the foremost acceptable treatment arrange and execute consistent with the sort of fault and connected info. In the end, the matter half are separated to create the grid work properly, reducing the user's troubles. The selfhealing system of the sensible installation will find and analyze its own faults during a bound amount, and update and optimize in real time, reducing the work of the employees.

Safe means that once the system is attacked by hackers, the sensible installation will resist and scale back the injury caused by the attack. within the power-based era, once the sensible installation has issues, several departments, units and even people are greatly affected, might which might \} be same that it's "A slight move in one half may have an effect on the case as a whole". High compatibility means during a sensible installation system, not solely centralized large- scale power plants however additionally several distributed power sources, power electronic elements and energy storage instrumentation, that square measure largely new energy sources. Finally, sensible coordination may be a sensible installation that covers a mature, complete power market operation with the goal of reducing resistance plugs and different constraints, and any linking to the wholesale and retail markets.

\subsection{Main risks of the smart power grid}

The main risks of the good grid square measure advanced, too frequent, used too wide, and too several terminals access. Nowadays, the event of science and technology in numerous fields is ever-changing with every passing day, that conjointly causes the quality of network communication. The protocols of assorted aspects like wireless network, mobile communication and satellite communication existing at a similar time ends up in the complicate state of affairs of good grid, which can cause info to be eavesdropped and destroyed within the transmission method. because the demand of users is obtaining larger and bigger, the frequency of data update is obtaining additional and additional frequent, the combination degree of assorted info is obtaining deeper and deeper, and also the communication between system and system, system and folks is additional frequent. an enormous quantity of data transmission will cause network instability, or perhaps crashed, and data is also destroyed and purloined at the terminal.

New technologies square measure used additional and additional wide, and new application directions have emerged altogether aspects like net of Things, wireless communication technology, virtualization, cloud computing, and AI technology. this case can step by step occur several security risks. The demand for intelligent terminals has exaggerated well, and business desires have conjointly exaggerated well, which can cause info lost or maliciously exploited. 


\section{CLOUD COMPUTING AND CLOUD COMPUTING IN SMART POWER GRID}

\subsection{Cloud computing}

Cloud computing is to gather an outsized quantity of extremely virtualized info to create an outsized resource pool. Users will use the resources within the resource pool to investigate and acquire solutions that area unit helpful to their own needs. This technology is a lot of convenient to manage and a lot of economical. The implementation of cloud computing technology needs 3 main architectures: the physical layer is that the most elementary structure of cloud computing, which might be same to be the basis of cloud computing; the virtual layer is to virtualize the data of the physical layer, and more distribute the calculation and space for storing of the physical layer moderately, that provides media for the operation of the cloud service; the cloud service manages and schedules the virtual layer to supply users with the desired services. With the support of cloud computing technology, users will access the desired information through the web, and also the information is centrally managed by the cloud server. Users solely have to be compelled to enter keywords to search out helpful info in an exceedingly great amount of sophisticated information. In essence, cloud computing is to place information, applications, and services within the cloud, creating full use of the labyrinthine virtual machines to create a automatic data processing system with supercomputing capabilities. Supported the characteristics of cloud computing, that area unit introduced into the good facility, a cloud service platform for the facility system is made to integrate this information the maximum amount as doable, and maximize the potential of information acquisition and data process of the facility.

\subsection{Cloud Computing in Smart Power Grids}

Use The information within the sensible facility is knotty, and also the users area unit hard-to-please a lot of and a lot of services, leading to Associate in Nursing exponential growth of information and also the ancient facility info is troublesome to satisfy the information storage. The distribution knowledge of sensible power grids is incredibly in depth, and also the knowledge to be provided incorporates a type of services and frequencies. additionally, the sensible facility conjointly needs an outsized range of calculations, analysis, simulation, improvement, style and deciding of the ability system. ancient power systems area unit troublesome to undertake such great deal calculations. For cloud computing, the strategy of classification and induction is employed to gather and organize knowledge. Of course, some strategies area unit accustomed maintain the safety of the system. Its knowledge management capability is adequate to manage all types of information within the sensible facility, so the system will handle totally different issues at an equivalent time, and improve the potency of the system. Moreover, cloud computing has programming capabilities that create calculations easier. In summary, applying cloud computing to a wise facility will solve a series of issues of enormous knowledge, difficult knowledge sorts, and troublesome knowledge analysis.

The sensible installation provides services to users with AN open pattern, distributes and monitors through virtualized computing, and provides users with power services that satisfy their desires. The design of the sensible installation is especially composed of 4 parts: physical storage layer, basic management layer, application interface layer, and advanced access layer. the ability connected system can unify all the valid information, so it will complement the shortcomings and supply services for the grid. At an equivalent time, as a result of their square measure additional and additional connected facilities within the sensible installation, there square measure additional and additional connected information. If we tend to solely place confidence in the interior cloud computing of the ability system, it's tough to satisfy the storage, calculation and analysis of the info. the event of sensible power grids is dynamic with every passing day, and users square measure progressively following the standard of services. Some folks have planned the thought of power grids cloud computing socialization for this development, and realized the safety of public clouds through access management and stratified management, which may maximize the usage in economy.

\section{APPLICATION MODE OF CLOUD COMPUTING IN SMART POWER GRID}

\subsection{Cloud Computing Intelligent Network Data Device}

Multi-directional transmission of power connected knowledge is that the application benchmark of good power system. Huge knowledge are going to be transmitted to every distribution. Once the relevant instrumentation needed by the grid is showing intelligence processed, these devices will method and analyze the info collected daily. The foundation of this approach is that the super knowledge storage of cloud computing, that is named cloud storage. the huge quantity of information generated by the user on a daily basis can enter the info central process library, and therefore the user doesn't ought to install the hardware to store the info, that greatly reduces the connected instrumentation investment, and therefore the user will get his own knowledge inadvisably once the safety verification. so as to enhance the safety issue and utilization of information keep within the cloud computing platform of the facility system, cloud computing typically uses a distributed storage technique to store knowledge, and multiple backups area unit wont to guarantee knowledge dependableness. Additionally, the cloud system conjointly give services for users, in order that the work of cloud computing is way larger. So as to satisfy such demands, cloud computing has to have a powerful capability to method knowledge from one place to a different.

In the future, the good installation will privatize some enterprises' cloud computing, and establish large-scale computing systems and cloud platform services in many regions to produce basic instrumentality and cloud computing service platforms to their various jurisdictions. At an equivalent time, so as to run the system often and 
expeditiously, the computing power is extended, however the authority to handle the business ought to be centralized. so as to shield enterprise information in any respect levels, cloud computing services rationally pool information from all levels of power corporations ANd established an data resource library at headquarters. In brief, the good installation information device design that introduces cloud computing consists of 3 parts: the resource layer covers varied resources, storage connected servers, tape libraries and a few connected application software; the center layer is that the most vital a part of cloud storage, that covers varied management modules like information, resources, data,operations, and security. Information management is to possess sure management over the flow of every information within the cloud computing setting. Resource management is to seek out \{the information theinfo the information $\}$ that's useful to users in massive data and classify them. data management is that some information within the cloud platform might cause harm to the system, and connected data management instrumentality ought to monitor and delete it; Task management refers to the management of storage tasks; Security management is security observation of cloud computing data, and also the user layer as a form of interface is that the carrier to produce services for users

\subsection{Cloud Computing Smart Power Grid Security Maintenance}

The responsiveness of cloud computing is without doubt powerful. once the ability system is subjected to varied attacks, cloud computing can resolve in time to confirm the safety of the system. Therefore, the institution of a cloud- based mostly good facility security maintenance platform is extremely necessary, which might not solely make sure the safety of the grid, however conjointly cut back the output of monetary and material resources. Cloud computing will maintain the safety of the system, however it conjointly must discover the information, thus a terminating station is ready up to transfer the problematic information collected by every branch enterprise to the central station for centralized process, and conjointly to move with the branch enterprises to seek out and solve the matter. the safety Maintenance Center is additionally composed of many totally different branches that employment along to confirm the safety of the system. These branches include: The consumer running cloud security maintenance module, that finds the matter templates collected by the virus unharness center, then compare it with the matter model collected by the information assortment center. If it's identical, then perform anti-virus and correction in time, or analyze the matter model of the information assortment center with the newest technology, insert the result into the matter model library, then publish by the virus commercial enterprise center to the consumer. At present, the foremost necessary strategy for maintaining the safety of the cloud platform is to severally manage and sight, victimization totally different levels protection, and increasing the amount of defense layers. The relevant service organization of the cloud platform must copy the user's information to confirm the reliableness of the information, set the information management authority, and therefore the user will read the information through the relevant permissions.

\section{CONCLUSION}

Applying cloud computing to the good installation may be a major focus of this power business, and it conjointly leads the longer term direction of the facility business. The normal good installation will not meet the storage and operation of huge knowledge. The appliance of cloud computing is that the key to resolve this drawback. Cloud computing will offer a lot of support for the event of good power grids. Making certain the safe operation of cloud computing systems is a chance to market the event of good power in China. the appliance of cloud computing technology within the good installation technology will build China's economic development more and might conjointly satisfy the daily wants of users, higher offer sensible facilitate for folks, and improve people's quality of life.

\section{REFERENCES}

[1] J. Jiang Daozhuo, Shen Tugang, Li Haixiang. The role and significance of standardization and standardization of basic information in the construction of smart grid Automation of Electric Power Systems, 2009(20):1-6. J. Clerk Maxwell, A Treatise on Electricity and Magnetism, 3rd ed., vol. 2. Oxford: Clarendon, 1892, pp.68-73.

[2] J. Wang Dewen, Song Yaqi, Zhu Yongli. Smart grid information platform based on cloud computing. Automation of Electric Power Systems, 2010(22): 7-12K. Elissa, "Title of paper if known," unpublished.

[3] J. Chen Jie, Zhang Yueyu. Research on the Application of Cloud Computing in Smart Grid and Its Security Issues.ZTE Communications,2012(6):17-21.

[4] J. Zhu Yafei, Gui Ning, Hu Wei. Framework Research and Security Analysis in Smart Grid Cloud Computing Mode.Communication World, 2016(22):162-163.

[5] J. Yu Yong, Lin Weimin, Deng Song. Cloud computing application and security research in smart grid.Information Network Security,2011(6):41-43.

[6] Kumar, P., Mathew, L., Shimi, S. L., \& Singh, P. (2016). Need of ICT for Sustainable Development of Power Sector. Proceedings of International Conference on ICT for Sustainable Development, 607614. doi:10.1007/978-981-10-0129-1_63.

[7] P. Kumar and V. Kumar, "Energy storage options for enhancing the reliability of Power system in the presence of Renewable Energy Sources," 2020 Second International Conference on Inventive Research in Computing Applications (ICIRCA), Coimbatore, India, 2020, pp. 1071-1076, doi: 10.1109/ICIRCA48905.2020.9183349. 